\title{
Incentive value and spatial certainty combine additively to determine visual priorities
}

\author{
K.G. Garner ${ }^{1,2}$ (D) H. Bowman ${ }^{1} \cdot$ J.E. Raymond ${ }^{1}$ \\ Published online: 9 October 2020 \\ (C) The Author(s) 2020
}

\begin{abstract}
How does the brain combine information predictive of the value of a visually guided task (incentive value) with information predictive of where task-relevant stimuli may occur (spatial certainty)? Human behavioural evidence indicates that these two predictions may be combined additively to bias visual selection (Additive Hypothesis), whereas neuroeconomic studies posit that they may be multiplicatively combined (Expected Value Hypothesis). We sought to adjudicate between these two alternatives. Participants viewed two coloured placeholders that specified the potential value of correctly identifying an imminent letter target if it appeared in that placeholder. Then, prior to the target's presentation, an endogenous spatial cue was presented indicating the target's more likely location. Spatial cues were parametrically manipulated with regard to the information gained (in bits). Across two experiments, performance was better for targets appearing in high versus low value placeholders and better when targets appeared in validly cued locations. Interestingly, as shown with a Bayesian model selection approach, these effects did not interact, clearly supporting the Additive Hypothesis. Even when conditions were adjusted to increase the optimality of a multiplicative operation, support for it remained. These findings refute recent theories that expected value computations are the singular mechanism driving the deployment of endogenous spatial attention. Instead, incentive value and spatial certainty seem to act independently to influence visual selection.
\end{abstract}

Keywords Attention - Prediction · Expectation · Reward - Incentive

\section{Introduction}

Humans are good at learning that specific sensory information, or cues, can predict subsequent events. For example, we learn quickly that hearing a siren on the left predicts a speeding emergency vehicle appearing from that direction, or that seeing a smile predicts a likely future opportunity to gain social approval. Knowledge about where and when new, important sensory information may appear or new reward opportunities may arise is only useful, however, if such knowledge can influence how cognitive mechanisms prioritise information representation for the eventual control of behaviour. Yet, our understanding of how learning and experience modify such prioritisation of visual signals, i.e. visual selection,

K.G. Garner

getkellygarner@gmail.com

1 School of Psychology, University of Birmingham, Birmingham, UK

2 Queensland Brain Institute (79), University of Queensland, St Lucia, QLD 4072, Australia remains incomplete. Particularly unclear is how multiple concurrent sensory cues, each associated with and therefore predictive of specific consequent outcomes, are combined to influence visual selection. A central tenet of many cognitive, computational, and neurobiological theories of visual selection (Buschman \& Kastner, 2015; see Itti \& Koch, 2001; Moore \& Zirnsak, 2017, for reviews), is that competition for high-level neural representation of external objects is flexibly biased by information pertaining to goal-directed outcomes. Indeed, multiple endogenous sources appear to exert parallel influences on visual selection (see Hutchinson \& TurkBrowne, 2012, for a review), even when learned information is antithetical to the current goals as defined by the task-set (see Awh et al., 2012, for a review). In the current study, we specifically seek to understand how selection biases that stem from learned associations between sensory cues and reward outcomes (incentive value) are combined with biases driven by associations between sensory cues and the probable location of task-relevant target information (spatial certainty).

Recently, a large number of studies have shown that visual selection can be biased by the learned predictiveness and incentive value signalled by sensory cues (see Le Pelley et al., 
2016, for a review). Such studies typically present stimuli in a simple selection task that probabilistically predict the reward magnitude available for correct performance, allowing those stimuli to become value associated. Then, in a subsequent visual selection task, these stimuli are presented as targets or distractors. The central finding is that visual selection performance is better or worse, respectively, compared to when such stimuli are absent (Anderson et al., 2011; Chelazzi et al., 2014; Raymond \& O'Brien, 2009; although see Sha \& Jiang, 2016). Convergent evidence from the behavioural (Le Pelley et al., 2016), neuro-cognitive (e.g. Barbaro et al., 2017; Hickey et al., 2010; Raymond \& O'Brien, 2009) and neuroeconomic (e.g. Dorris \& Glimcher, 2004; Platt \& Glimcher, 1999; Stănişor et al., 2013) literature also provides a convincing picture that value cues influence visual selection proportionally to their associated reward value, underscoring the role of prior experience.

Another large body of literature arising much earlier (in the 1980s) showed that sensory signals that predict where taskrelevant information might appear also as bias visual selection (Posner 1980). When centrally presented, symbolic cues (e.g., arrows) are highly predictive of a target's location (valid cues), response times (RTs) are faster and accuracy better compared to when spatial cues predict the wrong location (invalid cues). Central, symbolic spatial cues are often termed endogenous due to the assumption that their ability to bias visual selection largely stems from previously acquired and internally accessed knowledge. Our interest here is how endogenous (learned) spatial associations interact with other learned associations, specifically value associations to control visual selection. Strong evidence that spatial cueing effects depend on learning is that they scale with the reliability of the cue (Lanthier et al., 2015; Vossel et al., 2006, 2015), being stronger when cues are more predictive and weaker when predictability is low. Indeed, cueing effects appear to be a linear function of the certainty gained (in bits of information) by a spatial cue (Prinzmetal et al., 2015).

Although the issue of how multiple endogenous influences act on goal-directed visual selection has been previously addressed (Awh et al. 2012; Klink et al. 2014), it remains unclear how incentive value and spatial-certainty information provided by pre-target cues are combined. One possibility, the Expected Value Hypothesis, is that visual selection is biased by the relative expected value, i.e. incentive value multiplied by spatial certainty for each outcome. Derived from economic theory (Morgenstern \& Von Neumann, 1953), expected value is conventionally defined as reward magnitude multiplied by reward probability; in the context of selective attention in response to pre-target cues, such a computation would be the product of cue incentive value and spatial certainty. It is likely to be normalised across potential outcomes given the trial context (Padoa-Schioppa \& Assad, 2008). Evidence for such an operation would suggest that incentive value and spatial certainty are combined by a common, perhaps singular, mechanism to exert influence on visual selection. Support for this theory comes from the finding that human saccadic initiation times are more tightly correlated with the relative expected value of potential upcoming target locations than with either the spatial certainty for the target location or its incentive value alone (Milstein \& Dorris, 2007). Moreover, close correspondence has been shown between neurons that fire proportionally to the incentive values associated with sensory cues and those that change their response to correlate with the spatial certainty introduced by a directional cue, at least in macaque V1 (Stănişor et al., 2013). Indeed, this latter finding was interpreted as providing evidence for a singular neural mechanism able to re-weight incentive values across the visual scene on-the-fly by updating computation of expected values, given post-cue spatial probabilities.

A second alternative is the Additive Hypothesis, which posits incentive value and spatial certainty exert independent, as opposed to multiplicative, influences on visual selection. Origins for this idea stem from computational theories proposing that variation in physical salience within a scene (a saliency map) for different stimulus dimensions (e.g., colour, motion or orientation) are independently calculated, then weighted and summed to create a visual priority map (Zhao \& Koch, 2012). Indeed, empirical investigations support the notion of additive effects for the combination of salience information based on different features, and report that nonlinear combinations across feature dimensions arise only when there is overlap between the underlying saliency mechanisms (Nothdurft, 2000). Although suggestive of the possibility that incentive value and spatial certainty might combine additively to control selection, it remains unclear whether additivity could apply to non-physical features, such as learned associations.

However, preliminary support for this possibility has been reported by Stankevich and Geng (2014). They asked participants to detect as quickly and accurately as possible a simple target that could appear on the left or right within a coloured placeholder. In their experiment, placeholder colour indicated the magnitude of response-contingent rewards, as it does in the experiment we report here. However, they provided no explicit spatial cues as to target location. Instead, across blocks and without instruction, the probability of the target appearing on one side versus the other was varied. Greater performance benefits were observed when the target appeared in the more probable location and when that location corresponded to a high versus a low value placeholder. Critically, these benefits were additive, suggesting that incentive value and spatial certainty acted independently to influence visual selection, according to additive-factors logic (Sternberg, 1998). However, in their experiment, spatialcertainty was established over many trials, allowing predictions about target location to be generated well before each 
trial began and certainly in advance of location-specific incentive information. This may explain why incentive information provided an additive "top-up" effect. Such effects might not occur when location-specific incentive information is available first and spatial certainty cues providing task relevant information are presented subsequently. Accordingly, how two different endogenous cues (incentive and spatial) might be combined in humans when they are available only via new sensory information as each trial unfolds remains unknown.

To address this issue, we conducted two experiments that combined the methods of relevant previous studies to directly test these two hypotheses. As described in Fig. 2, on each trial, participants viewed two spatially separated coloured placeholders that served as incentive cues for $400-500 \mathrm{~ms}$; placeholder colour signalled the reward value for a subsequently presented letter target, should it appear in that placeholder and be correctly identified. Then, a central symbolic spatial cue was presented that signalled which placeholder would more likely encircle the upcoming target. Then, $400 \mathrm{~ms}$ after spatial cue onset, one letter briefly appeared in each placeholder. The task was to identify the target (as one of two possible letters) as fast and accurately as possible. Incentive value for the potential target location was always either high or low on every trial; however, the reliability of the spatial cue (spatial certainty) was varied between blocks. The Expected Value Hypothesis, implicating a multiplicative relationship between incentive value and spatial certainty, predicts an interaction of these two variables on performance (see Fig. 1). Specifically, it predicts a super-additive effect when spatial certainty is high and a sub-additive influence when it is low. In contrast, the Additive Hypothesis implies simple additivity of incentive value and spatial certainty. Evidence for this would be simple main effects on performance of incentive value and spatial certainty with no interaction effect. In Experiment 1, we varied spatial certainty over a wide range. In Experiment 2, rewards for correct responses diminished as RT lengthened, creating conditions favouring the use of an expected value mode of cue combination. Results were evaluated using a Bayesian model selection approach. To anticipate, we found in both Experiments that the influence of incentive value and spatial certainty remains additive across all tested levels of spatial certainty, arguing against the Expected Value Hypothesis.

\section{Experiment 1}

Five levels of spatial certainty were tested. Although it is conventional in attention-cueing experiments to use valid spatial cues on $80 \%$ and invalid cues on $20 \%$ of trials, we included conditions wherein the computation of spatial certainty is trivial, i.e., as the spatial cue approaches $100 \%$ validity. We

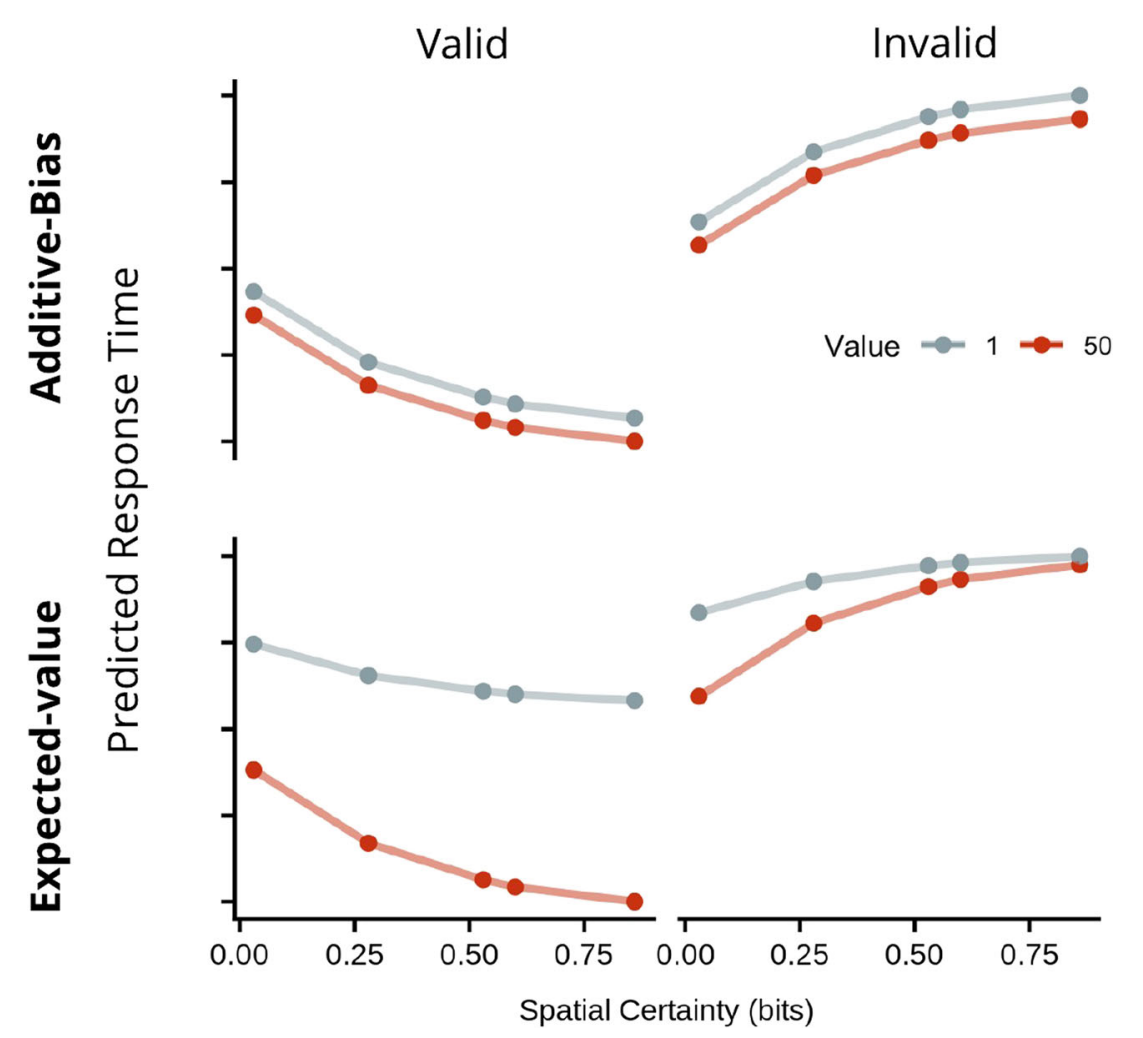

Fig. 1 Illustration of theoretical predictions. Predicted response times (RTs) in arbitrary units, plotted by cue validity (columns), spatial certainty (sc, $x$-axis) and incentive value (iv, colours), according to an additive bias operation $(\mathrm{y} \sim-\beta 1 \mathrm{sc}+-\beta 2 \mathrm{iv})$ or an expected-value model $(\mathrm{y} \sim$ $-\beta \mathrm{sc} * \mathrm{iv})$. (Note: The spatial certainties used in this simulation are the same as those chosen for Experiment 1) 
reasoned that if, as the Expected Value Hypothesis posits, a single mechanism responds to both incentive value and spatial-certainty information, then more resources would be available to respond to value information under high versus low spatial-certainty conditions, leading to greater versus lesser effects of incentive value, thereby showing super-additivity. To test this idea, we more densely sampled valid trial probabilities between .90 and .96 as well as the more conventional probabilities of .8 and .6 .

\section{Method}

All the task and analysis code and data from the current study are available online. ${ }^{1}$ The trial sequence of the spatialorienting task (Posner, 1980) used to assess the combined influence of spatial certainty and location-specific incentive cues, and the key manipulations for Experiments 1 and 2, are shown in Fig. 2.

\section{Participants}

As larger samples protect against spurious findings (Button et al., 2013; Lorca-Puls et al., 2018), we opted to double the sample size of previous work correlating human performance with expected value $(\mathrm{N}=10)$ (Milstein \& Dorris, 2007), and recruit a minimum of 20 participants. We calculated the stopping rule for data collection as the number of weeks where testing at maximum capacity would bring us to at least the minimum sample size ( 6 weeks with 4 people per week, allowing recruitment of $>20$ participants in order to protect against dropouts). Participants were recruited if they were aged 18 years or over and reported normal or corrected-tonormal vision, with no history of psychiatric or neurological illness, injury, or disorder. Participants earned either course credit or payment (£7 per session), and any additional rewards accrued during the session ( £10). All procedures were approved by the University of Birmingham Human Research Ethics Committee. A total of 23 participants were recruited. Of these, one was excluded due to technical failure and a second due to experimenter error. The remaining 21 participants (19 female, 18 right-handed, mean age $=20.3$ years, SD $4.5)$ completed all the procedures.

\section{Apparatus}

All experimental procedures took place in a room with a single testing station, under conditions of low ambient light. All tasks were programmed in Matlab (Mathworks, Natick, MA, USA, 2013a), using the Psychophysics Toolbox extension (Brainard, 1997; Pelli, 1997). The tasks were run on a Stone

\footnotetext{
${ }^{1}$ https://github.com/kel-github/attention-value-certainty
}

SOFREP-144 computer with a 23 -in. Asus VG278HE monitor $(1,920 \times 1,080$ pixels, $60-\mathrm{Hz}$ refresh rate) viewed from 57 $\mathrm{cm}$.

\section{Stimuli}

Two white (RGB: 200, 200, 200) vertical lines were presented in the centre of the screen. A darkening of one line $(50,50,50)$ served as the endogenous spatial cue. The entire spatial cue display (i.e. the rectangle comprising both lines and the grey background displayed between them) was $0.5^{\circ} \mathrm{w} \mathrm{x} 1^{\circ} \mathrm{h}$. Comparable cues have been used in previous work examining the influence of dynamic cue reliabilities on the deployment of spatial attention (Vossel et al., 2015), and therefore seemed appropriate for the aims of the current study. Although this type of central spatial cue had a small lateralised element, possibly activating exogenous orienting mechanisms to some extent, they were centrally presented (with $3.75^{\circ}$ between the outer edge of the central cue and the inner edge of the peripheral target), and, critically, require some interpretation from the brain to map the relationship between the cue and target locations (Berger et al., 2005; Briand \& Klein, 1987; Posner, 1980). Thus, the main characteristics of the cues used here resemble those used by previous researchers to study spatial certainty effects. Two coloured discs $\left(2.2^{\circ}\right.$ in diameter), one in purple $(87,75,80)$, the other in orange $(120,86,1)$ (matched for luminance) served as value cues. They were aligned along the horizontal meridian and positioned $4.5^{\circ}$ from the centre. Targets ('H' or ' $\mathrm{N}$ ') and distractors [' $\mathrm{Z}$ ' or ' $\mathrm{K}$ '] were presented in light grey $(90,90,90)$ Helvetica font, encompassed $1^{\circ}$, and were centred on the disc's centre. This distance from the centre allowed the viewer to discriminate the letters without making an eye movement, while remaining just within the locus of high-acuity vision $\left(\sim 6^{\circ}\right)$. Feedback was presented in green $(0$, $255,0)$ for high-reward values, amber $(255,191,0)$ for lowreward values, and red $(255,0,0)$ for errors. All stimuli were presented on a grey (RGB: 118, 118, 118) background.

\section{Procedure}

As shown in Fig. 2, each trial began with the simultaneous presentation of both incentive value cues and two centrally presented vertical lines. After a pseudo-randomly chosen duration of $400-500 \mathrm{~ms}$, the left or right fixation line darkened for $300 \mathrm{~ms}$. After a further $100 \mathrm{~ms}$, the target and distractor were presented for $100 \mathrm{~ms}$ (target identity was equiprobable for each incentive value $\mathrm{x}$ spatial certainty $\mathrm{x}$ cue validity condition). Participants pressed the ' $\mathrm{V}$ ' or ' $\mathrm{G}$ ' key on a standard keyboard to indicate the target identity. After $500 \mathrm{~ms}$, feedback was presented for $750 \mathrm{~ms}$; either the central fixation was replaced with the high-reward value, the low-reward value, an error signal (fixation lines turned red), or the fixation remained the same (no reward). Rewards were awarded on $80 \%$ of 


\section{a Incentive value} High Low

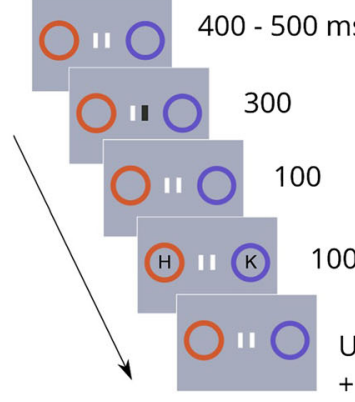

100 b

High reward Low reward

Experiment $1 \bigcirc 50 \bigcirc$

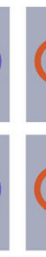

Experiment 2

Until response

$+250 \mathrm{~ms}$

C

Cue likelihood (Proportion valid/invalid)

\begin{tabular}{lccccc}
\hline & $.96 / .04$ & $.92 / .08$ & $.9 / .1$ & $.8 / .2$ & $.6 / .4$ \\
& Spatial-certainty (information gain in bits) \\
\hline Exp 1 & .86 & .6 & .53 & .29 & .029 \\
Exp 2 & & & & .29 & .029 \\
\hline
\end{tabular}

Feedback

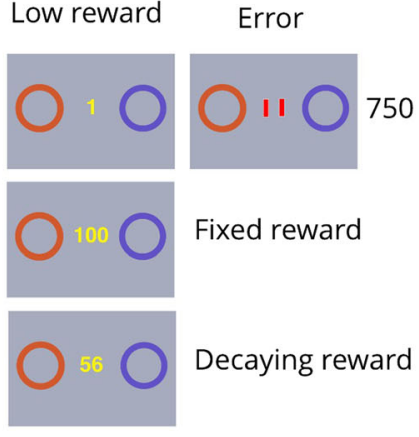

d

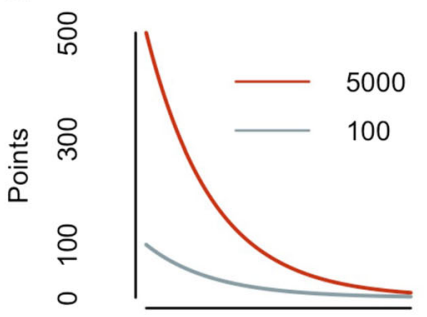

$\begin{array}{lll}0.0 & 0.4 \quad 0.8 \\ & \text { Time (s) }\end{array}$
Fig. 2 Study method. Task procedure and feedback conditions for Experiment 1 and Experiment 2. (a) Trial structure: Participants monitored two different-coloured circular placeholders (incentive cues). Colour indicated the magnitude of a performance-contingent reward for correct target ('H' or 'N') identification, should the target subsequently appear within that placeholder. Then, one of two central bars darkened, indicating the more likely target location (left, right). Note that the trial depicted is an invalid trial. (b) Reward feedback structure: After response $+250 \mathrm{~ms}$, performance feedback and response-contingent rewards were presented as shown. In Experiment 2, reward feedback was either independent of response time (fixed) or decremented exponentially after target onset until response (decaying). (c) Spatial certainty was parametrically manipulated across blocks by increasing the information gained (in bits) from the spatial cue. (d) Logic of the decaying reward condition in Experiment 2. Figure shows reward value available as a function of time from target onset. As the expected value computation involves a

correct trials to prevent feedback signals becoming redundant. The high- and low-incentive value cue locations and the target location were equally likely to be on the left or right; all conditions (cue value location, target location, and target identity) were fully crossed within each session. Colour/value pairings (e.g. purple $=50$ points $/$ orange $=1$ point) as well as targetresponse mappings were counterbalanced across participants.

Across blocks, the likelihood of cue validity was varied to be either .6 valid/.4 invalid, .8/.2, .9/.1, .92/.08, .96/.04, resulting in information gains (spatial-certainty) of $.029, .29$, $.53, .6$ and .86 bits. Each block contained 100 trials. At each of four sessions, participants completed four blocks for each level of spatial certainty. Participants took between 4 days and 1.5 weeks to complete the experiment (block order was pseudo-randomised for each session). Participants completed multiplicative weighting of spatial certainty and incentive value, responses should be super-additive or sub-additive depending on the spatial certainty/incentive value combination. As response times should reflect the inverse of this weighting, responses should be faster in a high-certainty/high-incentive scenario than responses based on an additive operation, and slower in a low-certainty/low-incentive scenario than an additive operation. Applying an exponential decay function to the incentive value at target onset means that the extra rewards accrued by being faster towards high-incentive value cues (change in the high $(5,000)$ value on the $\mathrm{y}$-axis, while moving leftward on the x-axis) would outweigh the losses accrued from being slower towards low-incentive value cues (change in the low (100) value on the $y$-axis, while moving rightward on the $x$-axis). Therefore, any operation that favours this response pattern would accrue greater total rewards than an additive operation, and therefore may emerge under such reward conditions

four sessions so that we could have sufficient trial numbers to obtain a reliable estimate of performance for the low spatialcertainty conditions. Target-value contingencies were split equally within each set of valid and invalid trials for each cue-likelihood condition.

Participants were explicitly instructed how many points were available should the target appear in the location of the high- and low-incentive value cues (50 vs. 1 point), and were instructed that the incentive cues signified that points were available most but not all of the time. They were also instructed that the darkened line was a clue to where the target could appear; however, they were not explicitly informed that the spatial cue's reliability might vary. Participants were requested to keep their eyes at fixation, and to respond as accurately and as quickly as possible to the target. Participants 
were also informed that their points would be exchanged for cash at the end of the session $(1,000=£ 1)$. At the start of the first session, participants practiced until they achieved at least $16 / 20$ correct responses.

\section{Statistical approach}

\section{Data pre-processing}

All data were analysed using the $\mathrm{R}$ programming language (v3.3.2) (R Core Team, 2013), and R Studio (v1.0.44) (RStudio Team, 2016). RT data were rejected if they were greater than $\pm 2.5 \mathrm{SD}$ from the mean for that participant in that condition. As participants were not explicitly informed when there was a change in spatial-certainty, we assumed that trials immediately subsequent to changes in spatial-certainty would be contaminated by learning effects. To remove the contaminated trials for each participant, we collapsed the data across spatial certainty blocks, and ordered the data according to trial number. We then fit piecewise linear regressions to find the break point that minimized the mean square error (MSE). Trials occurring prior to the breakpoint were removed $($ mean $=12.3$, SD 8.0). However, when we performed the analyses without removing these trials, the pattern of results was the same.

\section{Model specification and selection}

The aim of the study was to compare whether an additive model remained the best, given the data, even under conditions where an additive relationship could be expected to break down. The key aim of each analysis was to determine whether a model that included an interaction term between incentive value and spatial certainty was more probable, given the data, than one that only included main effects (i.e., an additive model). To quantify evidence, we used a Bayesian approach that provides the advantage of offering the ability to quantify evidence against a specific model, which is not possible using null hypothesis significance-testing approaches (Nickerson, 2000; Wagenmakers, 2007). Additionally, Bayesian approaches protect against the problem of model complexity: although more complex models may predict with high likelihood a greater range of values, if these predictions are uninformative, this will result in a more diffuse marginal likelihood distribution when integrating across prior distributions for the parameters, thereby penalising the resulting model evidence. First, we fit all possible linear mixed models on the RT data, with the regressors being (a) incentive value of the target location, (b) cue validity, and (c) spatial certainty offered by the cue. Spatial certainty was computed in line with Prinzmetal et al. (2015). Specifically, Shannon's (1948) measure of entropy $(\mathrm{H})$ measures the amount of uncertainty in a probability distribution and is at maximum when the cue is completely unpredictable with regard to the target location. Therefore, spatial certainty gained by an informative cue can be calculated as:

Spatial certainty $=\mathrm{H}_{\text {no information }}-\mathrm{H}_{\text {cue }}$

when $H$ is defined in the standard manner:

$\mathrm{H}=-\sum_{\mathrm{i}} \mathrm{p}_{\mathrm{i}} \log _{2} \mathrm{p}_{\mathrm{i}}$

and $p_{i}$ is the probability that the target appears at location $i$, given the cue. For example, with two locations, and a cue that is $.8 / .2$ valid/invalid:

$\mathrm{H}_{\text {cue }}=-\left(.8 \log _{2} .8\right)-\left(.2 \log _{2} .2\right) \approx .72$

As $H_{\text {no information }}$ is 1 (corresponding to complete uncertainty, i.e. .5/.5), then the information gained by the cue is $1-.72$ $\approx .28$ bits.

After fitting all possible models to the RT data obtained in each experiment, we computed Bayes factors (BFs) to quantify evidence for each linear mixed-effects model against the null model (intercept plus random effects of participant) using the Bayes factor package (Morey et al., 2014), and implementing the default Jeffreys-Zeller-Siow (JZS) prior on slope estimates (Liang et al., 2008). We then identified the six best performing models. We report the BF of the winning model relative to the null model, and the $\mathrm{BF}$ ratios between the best model and the next five best models, to demonstrate the strength of evidence in favour of the winning model. We follow the guidelines of Kass and Rafferty (Kass \& Raftery, 1995) when interpreting the strength of evidence. This was sufficient to determine whether the evidence favoured a model that included only main effects, or an incentive value $\mathrm{x}$ spatial certainty interaction. All BFs are reported along with the proportional error of the estimate. For readers interested in confirming that a null hypothesis significance testing (NHST) approach yields the same conclusions, please refer to the online repository for this study.

\section{Accuracy data}

Accuracy data were analysed to ensure the results were not due to a speed-accuracy trade-off. For each experiment, we fitted all possible linear mixed models to the accuracy data, and selected the winning model. We then reported the fixed effects estimates for each relevant factor in the winning model.

\section{Results}

\section{Response time}

Group mean RT data (dots) and winning model fit (lines) are presented in Fig. 3a. The main effect of incentive value was to 

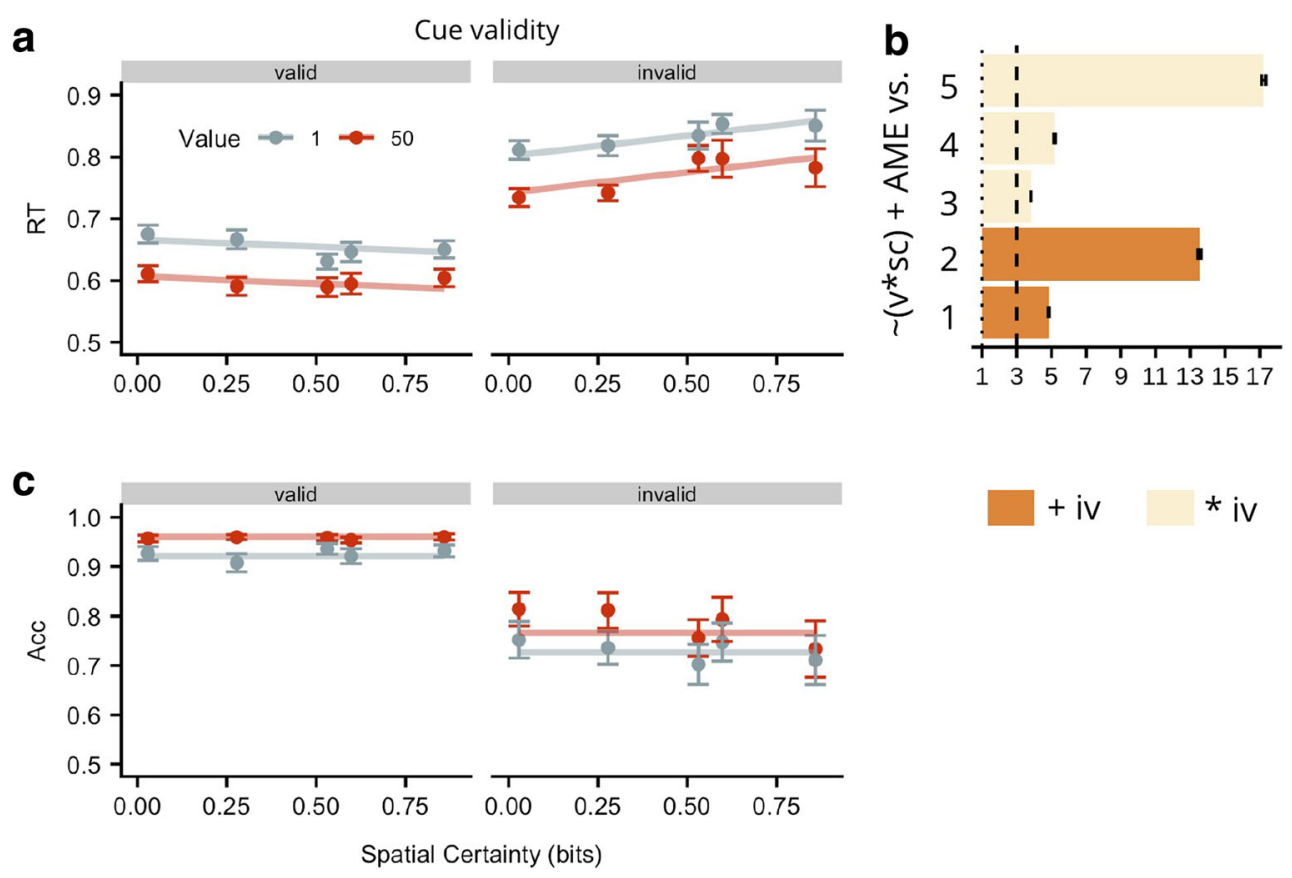

Fig. 3 Results from Experiment 1. (a) Observed group mean response times (RTs) in ms for targets appearing in high- (dark circles) or low(light circles) value placeholders plotted as a function of spatial certainty (x-axis) for valid and invalid spatial cues (panels). Vertical lines indicate \pm 1 within-subject SE. Lines represent fit of the winning model. The winning RT model did not involve any interaction of incentive value (iv) and spatial certainty (sc) supporting an Additive Hypothesis, although it did indicate an interaction of sc and validity (v). (b) BFs for the probability of the winning RT model (P[Win]: $(\mathrm{v} * \mathrm{sc})+\mathrm{AME})$ relative to the five next best models (Alternative, $\mathrm{P}[\mathrm{Alt}]$, models, $\mathrm{y}$-axis). The larger the BF value, the stronger the evidence for the winning model.

speed RT by approximately $50 \mathrm{~ms} \pm 3$ (SE) for high versus low incentives. Spatial certainty served to increase the effect of cue validity; the difference between valid and invalid trials increased by approximately $90 \mathrm{~ms} \pm 29$ (SE) across levels of certainty. Arguing against the Expected Value Hypothesis, the preferred model included only main effects of each factor (incentive value, spatial certainty, and cue validity), and a spatial certainty $\mathrm{x}$ cue validity interaction term $(\mathrm{BF}$, relative to the intercept only null model, $=1.74 \mathrm{E}+58, \pm .87 \%$; see Fig. $3 b)$. Importantly, there was positive evidence that this model was preferred over the next best model $(\mathrm{BF}=3.8, \pm 1.45 \%)$, which was identical to the winning model except that it also included an incentive value $\mathrm{x}$ spatial certainty interaction term. Therefore, the evidence favours a model that does not include an interaction between incentive value and spatial certainty.

\section{Accuracy}

The probability of an accurate response on invalid trials was $.16 \pm .02$ (SE) lower than on valid trials and was $.05 \pm .02$ (SE) less for targets appearing in low- versus high-incentive value
Any values lower than 1 (dotted line) support P[Alt]; BF values between 3 (dashed line) and 10 constitute moderate evidence for the winning model, values $>10$ provide strong evidence. Dark bars indicate P[Alt] contains only an additive influence of incentive value; light bars indicate $\mathrm{P}[\mathrm{Alt}]$ involves a multiplicative influence of incentive value and either spatial certainty or validity. The Alt RT models were as follows: (1) $\sim \mathrm{V}+$ iv, (2) AME, (3) $\sim(\mathrm{v} * \mathrm{sc})+(\mathrm{sc} *$ iv $)+\mathrm{AME},(4) \sim(\mathrm{v} * \mathrm{sc})+(\mathrm{v} * \mathrm{iv})+$ AME, $(5) \sim(\mathrm{v} * \mathrm{sc})+(\mathrm{v} * \mathrm{iv})+(\mathrm{sc} * \mathrm{iv})+\mathrm{AME}$. $($ c) Observed group mean accuracy plotted as in panel (a). Importantly, the accuracy data show that the RT findings were not due to a speed-accuracy trade-off. BF = Bayes factor, $\mathrm{rc}=$ reward condition, $\mathrm{AME}=$ all main effects.

cues. This shows that as participants became slower on invalid and low-incentive value trials, they also became less accurate, arguing against the notion that effects were due to a speed accuracy trade-off (see Fig. 3c). The preferred model for these data, relative to the null model, included only main effects of incentive value and cue validity $(\mathrm{BF}=8.83 \mathrm{E}+47, \pm .56 \%$, relative to the null model).

\section{Discussion}

There are two noteworthy findings from Experiment 1. First, even though the optimal strategy in this experiment would be to ignore the spatial cues entirely, especially in blocks with low spatial certainty, clear effects of valid versus invalid cues were found. Second, models posing an additive influence of incentive value and spatial certainty outperform those allowing an interaction between the two. This goes against the Expected Value Hypothesis and suggests that visual selection mechanisms do not integrate incentive value and spatial certainty, even when approaching the limits of certainty. Nevertheless, the second-best model to account for the RT 
data included an interaction between spatial certainty and incentive value, suggesting that this interaction is not entirely implausible. Perhaps additive effects would fail to provide the best description of the data if another form of appropriate pressure was applied to visual selection. Indeed, previous studies show that learning can direct the sampling of sensory information to optimise reward accrual (Drugowitsch et al., 2015; Kiani \& Shadlen, 2009; Serences, 2008), allowing the possibility that a reward structure favouring an expected value operation may be sufficient to modulate the additive influence of incentive value and spatial certainty. The aim of Experiment 2 was to provide this test.

\section{Experiment 2}

The aim of Experiment 2 was to test whether the additive influence found in Experiment 1 would persist even when the reward structure of the task renders additivity to be suboptimal. In Experiment 2, we leveraged the predictions made by the Additive and Expected Value operations to construct such a reward structure. As mentioned above, expected value computations are multiplicative, and should produce superadditive effects when both incentive values and spatial certainty are high, and sub-additive effects when incentive value and spatial certainty are low. Thus, RTs driven by an expected value operation should be faster than those driven by an additive operation when spatial certainty and incentive value are high and slower than an additive operation when spatial certainty and incentive value are low. Therefore, conditions that preferentially reward fast RTs on high-incentive, highcertainty trials and outweigh the costs incurred for slow RTs on low-incentive/low-certainty trials should favour adoption of an expected value strategy over an additive strategy (see Fig. 2d). To produce these reward conditions, in Experiment 2, participants completed the same task as in Experiment 1 (albeit sampling fewer levels of spatial certainty), with an added condition wherein reward value exponentially decayed after target onset. ${ }^{2}$ Of course, the assumption that these reward conditions favour a multiplicative (i.e. Expected Value) operation only holds if both spatial and incentive cues are leveraged to bias information processing. For example, an alternate and arguably optimal strategy would be to ignore the spatial cues entirely and to attend only to the high value location. However, we know from Experiment 1 that both cues effectively influence performance, making safe the assumption that decaying reward conditions favour a multiplicative over an additive operation.

\footnotetext{
${ }^{2}$ For a demonstration that participants were exposed to a sufficient range of the reward decay function to identify the reward structure, we refer the reader to the Online Supplementary Material: https://github.com/kel-github/ attention-value-certainty/blob/master/code-analysis-and-task/Supplement IncentiveValueAndSpatialCertainty.pdf
}

Replicating the finding from Experiment 1, evidence supports the Additive Hypothesis but not the Expected Value Hypothesis.

\section{Method}

\section{Participants}

We calculated the stopping rule for data collection as the number of weeks where testing at maximum capacity would bring us over the minimal sample size ( 3 weeks with 10 people per week). Of the 28 participants recruited, one was excluded due to technical difficulties with the eye tracker. A second participant was excluded as they did not meet the criterion required to terminate the practice. The remaining 26 (mean age $=19.5$ years, $\mathrm{SD}=1.03,24$ females, 26 righthanded) completed all the study procedures. Two of these participants had also completed Experiment 1.

\section{Apparatus}

In addition to that used for Experiment 1, an Eyelink $® 1000$ desktop-mounted eye-tracker (SR Research Ltd., Ottawa, Ontario, Canada) recorded movements of the left eye with a sampling frequency of $500 \mathrm{~Hz}$. This was used to ensure that eye movements were not contributing to results, even though participants were clearly instructed not to move their eyes (replicating instructions used in Experiment 1, which were not, however, verified for compliance).

\section{Stimuli}

The stimuli were the same as in Experiment 1, except that the value cues were presented at 5.7. This change was made to match the exact layout used in previous work (Stankevich \& Geng, 2014).

\section{Procedure}

The procedure was the same as Experiment 1 with the following exceptions. Participants' eyes were monitored on every trial. Calibration and validation were performed every 25 trials to minimise drift errors. If the participant's eyes moved more than 50 pixels (1.5) from the fixation at cue-offset, text appeared to notify participants they had been "too fast". The trial was then terminated. Terminated trials accounted for $\sim 3 \%$ of all trials.

Cue-values were increased from Experiment 1 to 5,000 versus 100 points, so that participants could gain at least 1 point when a decay was applied to the low incentive value. Correct responses were rewarded on $80 \%$ of trials as in Experiment 1. Given that there were 200 trials per 
condition (see below), and assuming $\sim 85 \%$ accuracy, this provides $\sim 136$ rewarded trials per condition. Previous studies suggest that participants can detect dynamics in incentive value associations with far fewer trials $(\mathrm{N}=8$, Ittipuripat et al., 2015) or lower reward-to-non-rewarded ratios (33:77\%, Serences, 2008), and that these detections influence visual spatial priorities. In the decay rewardcondition, an exponential decay function (reward value $=$ points $*\left(e^{-4 * R T}\right), \mathrm{RT}=$ Response Time) was applied to each value at target onset. The monetary value of points was adjusted so that participants received the same rate of cash payments as Experiment $1(100,000=£ 1)$. Participants were informed at the start of the decay blocks that the value available to them would begin to run out upon appearance of the target.

Participants completed 200 trials for each of four spatial certainty/reward contingency conditions (.29/fixed, .29/decay, .029/fixed, .029/decay; block order was counterbalanced across participants). Although trial number per condition was substantially lower than in Experiment 1, adjustments to changes in spatial certainty in other cuing studies have shown clear effects with less than half the trial numbers we used here (Prinzmetal et al., 2015; Vossel et al., 2015). We included only these two levels of spatial certainty as we wanted to avoid any possible floor or ceiling effects when testing the influence of reward condition.

We also tested the separate hypothesis that individuals may mentally represent the high- and low-incentive placeholders differently in terms of their relative value, when their value can be obtained more reliably (i.e. in the fixed reward-condition, relative to the decay reward-condition), and that this may be expressed via physical placement on a linear space. Every 50 trials, participants were instructed to use a mouse to drag the two placeholders wherever they liked on a single line. However, we found no evidence that cue-likelihood influenced placement of the placeholders $(p=.96)$, and this separate aspect of the study is discussed no further. Participants also completed a BIS/BAS questionnaire (Carver \& White, 1994) that was used to test a hypothesis for a separate study not reported here.

\section{Statistical approach}

We followed the same data-cleaning procedures as in Experiment 1. Again, piecewise linear functions were fitted to the data to isolate the trials contaminated by spatial certainty learning effects. The number of trials removed from the start of each block were similar to Experiment 1 (mean $=14.7, \mathrm{SD}$ 8.5).

We also used the same model comparison approach, with the exception that we added the reward condition term to the linear mixed effects models that were fit to the data.

\section{Results}

\section{Response time}

Group mean RT data are shown in Fig. 4a. Correct responses were approximately $21 \mathrm{~ms} \pm 17$ (SE) slower for targets appearing in low- versus high-incentive value placeholders; valid versus invalid spatial cues speeded RTs on average by $33 \mathrm{~ms} \pm 12$ (SE); and the decay-reward condition speeded RTs by $54 \mathrm{~ms} \pm 17$ (SE) on average compared to the fixed-reward condition. Unlike Experiment 1, there was no detectable influence of spatial certainty. (Note: We tested whether this could be attributed to a reduced sensitivity owing to the fewer levels of spatial certainty used in Experiment 2 relative to Experiment 1, by taking the data from Experiment 1 and extracting only the levels of spatial certainty that were used in Experiment 2). A repeated-measures ANOVA (incentive value $\mathrm{x}$ spatial certainty $\mathrm{x}$ cue validity) showed a significant spatial certainty $\mathrm{x}$ cue interaction $(\mathrm{F}(1,20)=4.84, \mathrm{MSE}=$ $.001, \mathrm{p}=.047$ ), suggesting that reducing the levels of spatial certainty is not sufficient to reduce detectability of such an interaction). As in Experiment 1, we identified the most likely model given the data and found that the winning model included main effects of cue validity, incentive value, and reward condition $(\mathrm{BF}=2.18 \mathrm{E}+67 \pm .69 \%$, relative to null model), but did not include an influence of spatial certainty (although see Accuracy data). There was good evidence that this was the best model for the data, as it was positively preferred to the next best model, which included an additional incentive value $x$ cue validity interaction term $(B F=4.65 \pm 2.43 \%$, see Fig. 4b). As spatial certainty was found to interact with cue validity in Experiment 1, we tested the evidence for the winning model against one that also included a spatial certainty $\mathrm{x}$ cue validity interaction term. Again, there was positive evidence that the winning model provided a better fit to the data $(\mathrm{BF}=8.97 \pm 1.79 \%)$. Collectively, the results show that even when an additive operation is disadvantageous, an additive model is still a better account of the data.

1) $\sim(\mathrm{rc} \mathrm{x}$ iv) $+\mathrm{v}+\mathrm{iv}+\mathrm{rc}, 2) \sim \mathrm{AME}, 3) \sim(\mathrm{rc} \mathrm{x} \mathrm{v})+\mathrm{iv}+\mathrm{v}+$ $\mathrm{rc}, 4) \sim(\mathrm{v} \mathrm{x} \mathrm{sc})+\mathrm{AME}, 5)(\mathrm{v} x \mathrm{iv})+\mathrm{v}+\mathrm{iv}+\mathrm{rc}$. BF $=$ Bayes factor, $\mathrm{v}=$ cue-validity, $\mathrm{sc}=$ spatial certainty, $\mathrm{iv}=$ incentive value, $\mathrm{rc}=$ reward condition, $\mathrm{AME}=$ all main effects

\section{Accuracy}

Differences in accuracy between valid and invalid trials grew larger as spatial certainty increased (by .15 on average, \pm .07 (SE)), suggesting that as participants became slower on invalid trials, they also became less accurate. Furthermore, accuracy performance was slightly higher when targets appeared in high- relative to low-incentive value placeholders (by approximately $.0003, \pm .0001$ (SE)), showing that participants became very modestly less accurate as they slowed their 


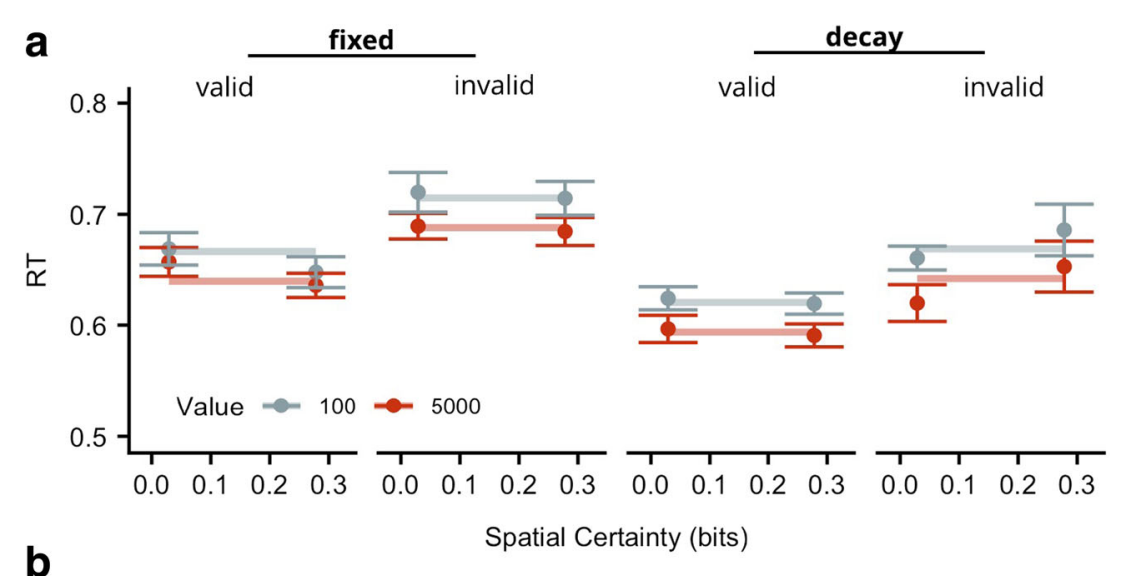

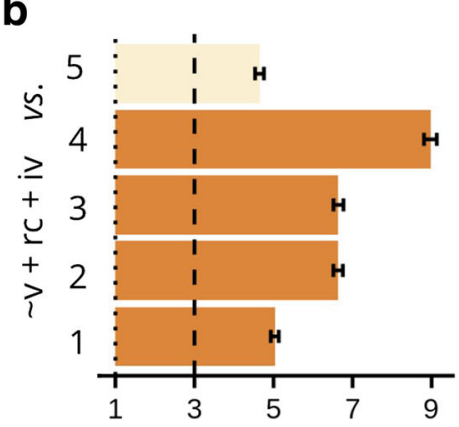

Fig. 4 Response time (RT) Experiment 2. (a) Observed group mean RTs in ms for targets appearing in high- (dark circles) or low- (light circles) value placeholders plotted as a function of spatial certainty (x-axis) for valid and invalid spatial cues (panels) for each reward condition. Vertical lines indicate \pm 1 within-subject SE. Lines represent fit of the winning model. The winning model involved main effects of incentive value, cue validity and reward condition. (b) BFs for the probability of the winning RT model (P[Win]: $\mathrm{v}+\mathrm{rc}+\mathrm{iv})$, relative to the five next best models (Alternative, $\mathrm{P}[\mathrm{Alt}]$, models, $\mathrm{y}$-axis). The larger the $\mathrm{BF}$ value, the stronger

responses to low-value targets. Accuracy was higher for the fixed relative to the decay reward condition $(.05, \pm .008$ (SE)), suggesting that as participants became faster in the delayed reward condition, they also produced a very modest decrease in accuracy. As in Experiment 1, the accuracy data obtained here demonstrate that the validity and incentive value results were not due to a speed-accuracy trade off, whereas the influence of reward condition may indeed be due to such a tradeoff (see Fig. 5). The best-fitting model to account for these data contained a cue-validity $\mathrm{x}$ spatial certainty interaction, and main effects of incentive value, spatial certainty and cue validity.

\section{General discussion}

Over two experiments we tested whether the Additive Hypothesis would outperform the expected value account, even under conditions expected to challenge the optimality of additivity. In Experiment 1, we hypothesised that if incentive value and spatial certainty influence a common underlying mechanism, then conditions wherein spatial certainty is evidence for the winning model. Any values lower than 1 (dotted line) support $\mathrm{P}[\mathrm{Alt}]$. The larger the BF value, the stronger the evidence for the winning model. Any values lower than 1 (dotted line) support P[Alt]; BF values between 3 (dashed line) and 10 constitute moderate evidence for the winning model, values $>10$ provide strong evidence. Dark bars indicate P[Alt] only contains an additive influence of incentive value; light bars indicate P[Alt] involves a multiplicative influence of incentive value and either spatial certainty or validity. The Alt RT models were as follows:

trivial to compute (i.e., very high certainty) might best reveal non-additive effects, because these conditions should be minimally taxing to central resources and thus be more likely to enable an influence of incentive value on visual selection. We created this condition by using very high spatial-certainty cues and then pitted incentive value and spatial certainty against each other in a spatial-orienting task, where endogenous cues signalled the likely location of upcoming letter targets. Interestingly, an additive influence of incentive value and spatial certainty was observed, even under conditions of very high certainty. Spatial certainty increased the size of the cueing-effect (i.e. the difference in performance between invalidly and validly cued trials), whereas incentive value had a comparable influence on both valid and invalid trial types, across all levels of spatial certainty.

In Experiment 2, we reasoned that if an expected value operation can bias visual selection, then a reward structure that favours a multiplicative weighting of incentive value and spatial certainty may reveal it. We applied an exponential decay function to incentive values at target onset; this ensured that if RTs were driven by multiplicative as opposed to additive weighting, then reward gains accrued by faster RTs under 


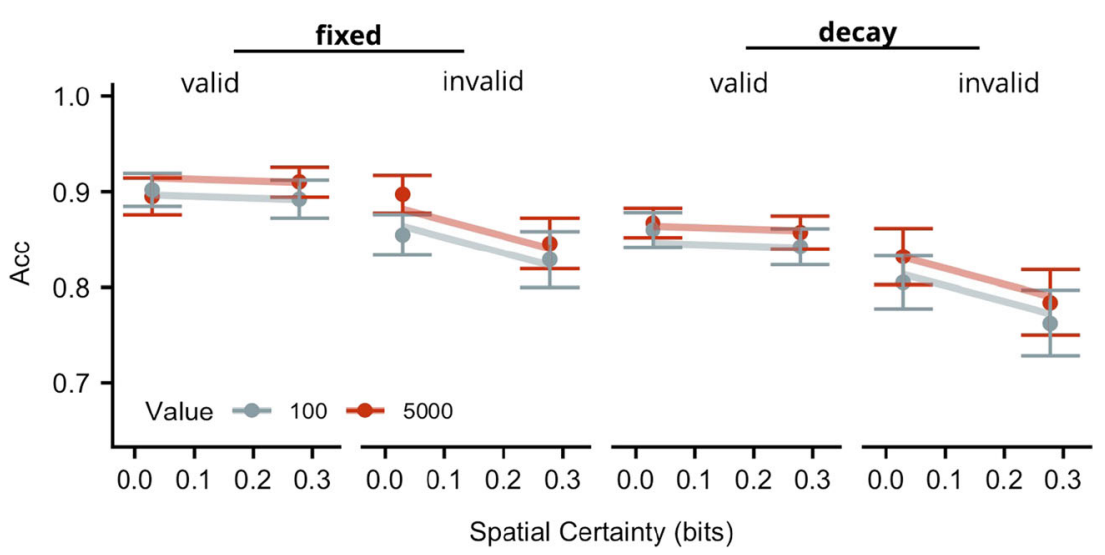

Fig. 5 Accuracy data for Experiment 2. Accuracy (Acc) for targets appearing in high- (dark circles) or low- (light circles) value placeholders plotted as a function of spatial certainty (x-axis) for valid and invalid

high-incentive value/high-certainty conditions would outweigh losses incurred by slower RTs under low-incentive value/low-certainty conditions, relative to RTs predicted by an additive model. Although the influence of spatial certainty manifested differently to Experiment 1, i.e., by modulating accuracy, rather than RT, we observed that the effect of incentive value remained additive to spatial certainty and to other experimental factors. Again, the findings support the Additive Hypothesis. However, this interpretation depends on the assumption that participants were able to infer the relationship between RT and rewards. Although participants were both explicitly informed about the decaying reward and were exposed to a sufficient range of the function to theoretically be able to infer the relationship between RT and rewards, an explicit measure of the participant's understanding of the $\mathrm{RT} /$ reward relationship should be taken in future studies to verify this assumption.

What kind of mechanism or computation could result in a robust additive influence between incentive value and spatial certainty? In concert with recent theoretical and empirical developments suggesting that cognitive control processes are offset by subjective and computational costs of effortful control (Braver, 2012; Yee \& Braver, 2018), we believe the current data can be interpreted as reflecting trial-by-trial adaptations aimed at the conservation of effort. If we assume that the maintenance of the task set, i.e., a priori preparedness to identify a data-limited target at two locations, requires energetic resources from the underlying selection mechanism, it is of benefit to the brain to predict conditions where effort can be relaxed, in order to conserve energy expenditure. For example, by learning the energetic range over which target identification mechanisms can be adjusted, to ensure good enough target detection, given the task parameters. According to this view, a cost-benefit analysis could inform how much energy could be saved, given an acceptable decrement to accuracy and RT.

Applied to the current context, after the onset of the incentive value cues, selection mechanisms should maintain a spatial cues (panels) for each reward condition. Vertical lines indicate \pm 1 within-subject SE. Lines represent fit of the winning model

steady level of task preparation favouring the high-value location, for example, by increasing the excitability of neuronal assemblies whose collective receptive fields correspond to detecting lines or edges at that location (Carrasco, 2011; Desimone \& Duncan, 1995; Roelfsema et al., 2000; Schmitz \& Duncan, 2018), thereby biasing the system towards a stronger response to the upcoming stimulus (Buschman \& Kastner, 2015). Concurrently, the excitability of neuronal assemblies directed towards encoding information from the low-value location should be relaxed, as the cost of sometimes missing the target at that location, given the energy needed to detect it, should become negligible. Similarly, upon spatial cue onset, preparation of such target detection mechanisms could be further relaxed for the unlikely location, proportionally to how unlikely that location is to possess a target. Importantly, this is performed incrementally to the previous adjustment. This interpretation suggests that the system incrementally updates cost-efficient sensory encoding on the basis of incoming information. This interpretation predicts that the degree to which incentive value or spatial certainty can influence performance is dependent upon the range over which preparatory processes can be titrated and still yield acceptable performances. This would account for why, in Experiment 2, under a context with greater pressure on RT performance, we observed an influence of cue validity but not spatial certainty. Presumably, it had become too costly to modulate RT performance by spatial certainty and meet the perceived demands of the task.

The current findings shed further insights into the previously proposed unitary selection mechanism that biases competition between cortical representations of stimuli, in the presence of both incentive and explicit spatial cues (Stănișor et al., 2013). These authors showed that overlapping clusters of macaque V1 neurons were sensitive to both incentive value cues and $100 \%$ valid spatial cues. The authors proposed that the explicit spatial cue served to re-weight the relative value between the target and distractor, and that this re-weighting was instantiated by a unitary selection mechanism that computes 
the relative expected value between targets. The current study shows that the spatial certainty derived from explicit cues does not contribute to an expected value operation with the previous incentive values to re-weight the relative value between the two items. Rather, an additive influence points to the repeated invocation of a selection mechanism on the basis of updates from separable information sources. As incentive value and spatial certainty appear to have been added, rather than multiplied, to the existing output of the selection mechanism, the two sources of information may be transformed into a common representational space, or unit, prior to the enactment of their influence. ${ }^{3}$

An alternate, but not mutually exclusive, reason for the independence of incentive value and spatial certainty may be that the two are governed by different learning systems; for example, recent investigations measuring overt eye movements, rather than covert attention shifts, found that incentive values induce associative behaviours (Kim \& Anderson, 2019 ; i.e. participants look at the value cue even when paid not to; Le Pelley et al., 2015), whereas spatial cues may be learned via instrumental conditioning (i.e. participants are more likely to repeat an orienting behaviour that has been reinforced; Kim \& Anderson, 2019). Indeed, evidence that visual selection biases remain present when explicit spatial cues (i.e. arrows or words) are non-predictive suggests that these behaviours may be under the control of the habitual learning system (Jiang, 2018; Y in \& Knowlton, 2006), which governs learned stimulus-response contingencies. Moreover, in the current experiments, we have taken a snapshot of the relationship between incentive value and spatial certainty at one time point (400 ms stimulus-onset asynchrony (SOA)), potentially when the system is still in a state of updating. It is possible that a multiplicative relationship between spatial certainty and incentive value emerges later in time, once the

\footnotetext{
${ }^{3}$ An alternate explanation for the observed additive relationship between spatial certainty and incentive value on RT performance is that an expected value operation is being performed by summation on a logarithmic scale. For example, the expected utility of the scene $(E(U))$ could be computed as follows:

$$
\mathrm{E}(\mathrm{U})=(\operatorname{SC}(\mathrm{T}) * \operatorname{IV}(\mathrm{T}))+(\operatorname{SC}(\mathrm{D}) * \operatorname{IV}(\mathrm{D}))
$$

and

$$
\mathrm{E}^{\prime}(\mathrm{U})=\log \mathrm{E}(\mathrm{U})
$$

where SC is spatial certainty, IV is incentive value, $\mathrm{T}$ is the target location and $\mathrm{D}$ is the distractor location. In this case, if the distractor location's priority was assigned a value of zero, $\mathrm{E}(\mathrm{U})$ would reduce to $\log (\mathrm{SC}(\mathrm{T}))+$ $\log (\operatorname{IV}(\mathrm{T}))$, i.e., an additive relationship between spatial certainty and incentive value. However, such an account hinges on the notion that the distractor location is somehow assigned a value of zero. This interpretation seems unlikely given that the distractor location has value, and can never be fully ruled out as a potential target location. Moreover, such a computation should produce logarithmic, and not linear, relationships between spatial certainty and RTs, when the latter was observed across both Experiment 1 and Experiment 2.
}

information from each cue has undergone further processing. This is a possible avenue of further investigation in future studies.

An additive influence of incentive value on visual selection was also observed by Stankevich and Geng (2014), when value was pitted against the varying probability that a target would appear on one side versus the other, in the absence of explicit spatial cues. A visual comparison of the current data and the data from Stankevich and Geng (2014) also yields some interesting points of difference concerning the influence of spatial certainty in the presence or absence of explicit spatial cues. With the current explicit cues, we observe RTs that are comparable across spatial-certainty conditions for valid cues, whereas RTs on invalid trials increase as spatial certainty decreases. We inspected the previous literature and observed that this is a consistent finding across other studies that varied the probabilities of explicit spatial cues in comparable tasks (Lanthier et al., 2015; Vossel et al., 2006), suggesting that this is a replicable phenomenon, rather than the consequence of a ceiling effect or something similar. In contrast, Stankevich and Geng (2014) observed the opposite: RTs decreased as target location became more likely (analogous to valid trials) but remained invariant when directed towards increasingly less likely (invalid) locations. Therefore, our results suggest that the explicit spatial cue we used resulted in preparation towards the cued location that did not vary with the certainty offered by the cue, coupled with a relaxation of preparation towards the invalid location that scaled with certainty. In contrast, non-explicit spatial knowledge appears to cause a strengthening of preparation towards the more likely location, with no concomitant relaxation towards the unlikely location. This suggests that spatial certainty influences visual selection differently dependent on how it is learned. For example, a long history of arrows serving as useful directional cues could motivate a strong response to the directional stimulus, against which other useful behaviours can be adapted. Therefore, it appears that visual selection behaviours adapt to environmental information in relation to the most contextually relevant baseline behaviour.

\section{Conclusions}

Over two experiments, we sought to arbitrate between competing theories for how learned associations pertaining to incentive value and spatial certainty combine to influence visual selection. Specifically, we asked whether this influence was additive (Additive hypothesis), or multiplicative (Expected Value hypothesis). We tested these hypotheses by pitting incentive values and spatial certainties against one another in a spatial cueing task under conditions expected to challenge the optimality of an additive operation. The data from two experiments support the notion that visual selection mechanisms show independent sensitivity to incentive value and spatial- 
certainty information, and that both information sources are converted to a common representational space, or unit, in order to influence visual selection. We also interpret our results as suggesting that the mechanisms underpinning visual selection dynamically leverage distinct information sources to reflexively conserve effort within a range that allows acceptable performance given the current task parameters.

Acknowledgements K.G. Garner would like to acknowledge Christopher Nolan, Andrew Clouter and Dragan Rangelov for their comments and insightful discussions about this work. This project was supported by grant ES/L000210/1 from ESRC to J Raymond. K.G. Garner is also supported by a Marie Sklodowska Curie Global Fellowship.

The authors declare no competing interests.

Open Access This article is licensed under a Creative Commons Attribution 4.0 International License, which permits use, sharing, adaptation, distribution and reproduction in any medium or format, as long as you give appropriate credit to the original author(s) and the source, provide a link to the Creative Commons licence, and indicate if changes were made. The images or other third party material in this article are included in the article's Creative Commons licence, unless indicated otherwise in a credit line to the material. If material is not included in the article's Creative Commons licence and your intended use is not permitted by statutory regulation or exceeds the permitted use, you will need to obtain permission directly from the copyright holder. To view a copy of this licence, visit http://creativecommons.org/licenses/by/4.0/.

\section{References}

Anderson, B. A., Laurent, P. A., \& Yantis, S. (2011). Value-driven attentional capture. Proceedings of the National Academy of Sciences of the United States of America, 108(25), 10367-10371. https://doi. org/10.1073/pnas.1104047108

Awh, E., Belopolsky, A. V., \& Theeuwes, J. (2012). Top-down versus bottom-up attentional control: a failed theoretical dichotomy. Trends in Cognitive Sciences, 16(8), 437-443. https://doi.org/10.1016/j. tics.2012.06.010

Barbaro, L., Peelen, M. V., \& Hickey, C. (2017). Valence, Not Utility, Underlies Reward-Driven Prioritization in Human Vision. The Journal of Neuroscience: The Official Journal of the Society for Neuroscience, 37(43), 10438-10450. https://doi.org/10.1523/ JNEUROSCI.1128-17.2017

Berger, A., Henik, A., \& Rafal, R. (2005). Competition between endogenous and exogenous orienting of visual attention. Journal of Experimental Psychology. General, 134(2), 207-221. https://doi. org/10.1037/0096-3445.134.2.207

Brainard, D. H. (1997). The Psychophysics Toolbox. Spatial Vision, 10(4), 433-436. http://www.ncbi.nlm.nih.gov/pubmed/9176952

Braver, T. S. (2012). The variable nature of cognitive control: a dual mechanisms framework. Trends in Cognitive Sciences, 16(2), 106-113. https://doi.org/10.1016/j.tics.2011.12.010

Briand, K. A., \& Klein, R. M. (1987). Is Posner's "beam" the same as Treisman's "glue"?: On the relation between visual orienting and feature integration theory. Journal of Experimental Psychology. Human Perception and Performance, 13(2), 228-241. https://doi. org/10.1037/0096-1523.13.2.228

Buschman, T. J., \& Kastner, S. (2015). From Behavior to Neural Dynamics: An Integrated Theory of Attention. Neuron, 88(1), 127-144. https://doi.org/10.1016/j.neuron.2015.09.017
Button, K. S., Ioannidis, J. P. A., Mokrysz, C., Nosek, B. A., Flint, J., Robinson, E. S. J., \& Munafò, M. R. (2013). Power failure: why small sample size undermines the reliability of neuroscience. Nature Reviews. Neuroscience, 14(5), 365-376. https://doi.org/10.1038/ nrn3475

Carrasco, M. (2011). Visual attention: the past 25 years. Vision Research, 51(13), 1484-1525. https://doi.org/10.1016/j.visres.2011.04.012

Carver, C. S., \& White, T. L. (1994). Behavioral inhibition, behavioral activation, and affective responses to impending reward and punishment: The BIS/BAS Scales. Journal of Personality and Social Psychology, 67(2), 319. https://doi.org/10.1037/0022-3514.67.2. 319

Chelazzi, L., Eštočinová, J., Calletti, R., Lo Gerfo, E., Sani, I., Della Libera, C., \& Santandrea, E. (2014). Altering spatial priority maps via reward-based learning. The Journal of Neuroscience: The Official Journal of the Society for Neuroscience, 34(25), 8594 8604. https://doi.org/10.1523/JNEUROSCI.0277-14.2014

Desimone, R., \& Duncan, J. (1995). Neural mechanisms of selective visual attention. Annual Review of Neuroscience, 18, 193-222. https://doi.org/10.1146/annurev.ne.18.030195.001205

Dorris, M. C., \& Glimcher, P. W. (2004). Activity in posterior parietal cortex is correlated with the relative subjective desirability of action. Neuron, 44(2), 365-378. https://doi.org/10.1016/j.neuron.2004.09. 009

Drugowitsch, J., DeAngelis, G. C., Angelaki, D. E., \& Pouget, A. (2015). Tuning the speed-accuracy trade-off to maximize reward rate in multisensory decision-making. eLife, 4, e06678. https://doi.org/10. 7554/eLife.06678

Hickey, C., Chelazzi, L., \& Theeuwes, J. (2010). Reward changes salience in human vision via the anterior cingulate. The Journal of Neuroscience: The Official Journal of the Society for Neuroscience, 30(33), 11096-11103. https://doi.org/10.1523/ JNEUROSCI.1026-10.2010

Hutchinson, J. B., \& Turk-Browne, N. B. (2012). Memory-guided attention: control from multiple memory systems. Trends in Cognitive Sciences, 16(12), 576-579. https://doi.org/10.1016/j.tics.2012.10. 003

Itti, L., \& Koch, C. (2001). Computational modelling of visual attention. Nature Reviews. Neuroscience, 2(3), 194-203. https://doi.org/10. 1038/35058500

Itthipuripat, S., Cha, K., Rangsipat, N., Serences, J.T. (2015). Valuebased attentional capture influences context-dependent decisionmaking. J Neurophys, 1:560-9

Jiang, Y. V. (2018). Habitual versus goal-driven attention. Cortex; a Journal Devoted to the Study of the Nervous System and Behavior, 102, 107-120. https://doi.org/10.1016/j.cortex.2017.06.018

Kass, R. E., \& Raftery, A. E. (1995). Bayes Factors. Journal of the American Statistical Association, 90(430), 773-795. https://doi. org/10.2307/2291091

Kiani, R., \& Shadlen, M. N. (2009). Representation of confidence associated with a decision by neurons in the parietal cortex. Science, 324(5928), 759-764. https://doi.org/10.1126/science.1169405

Kim, H., \& Anderson, B. A. (2019). Dissociable Components of Experience-Driven Attention. Current Biology: CB, 29(5), 841845.e2. https://doi.org/10.1016/j.cub.2019.01.030

Klink, CP., Jentgens, P., \& Lorteije JAM. (2014). Priority Maps Explain the Roles of Value, Attention, and Salience in Goal-Oriented Behavior. J Neurosci, 34(42):13867-13869. https://doi.org/10. 1523/JNEUROSCI.3249-14.2014

Lanthier, S. N., Wu, D. W.-L., Chapman, C. S., \& Kingstone, A. (2015). Resolving the controversy of the proportion validity effect: Volitional attention is not required, but may have an effect. Attention, Perception \& Psychophysics, 77(8), 2611-2621. https:// doi.org/10.3758/s13414-015-0956-8

Le Pelley, M. E., Mitchell, C. J., Beesley, T., George, D. N., \& Wills, A. J. (2016). Attention and associative learning in humans: An 
integrative review. Psychological Bulletin, 142(10), 1111-1140. https://doi.org/10.1037/bul0000064

Le Pelley, M. E., Pearson, D., Griffiths, O., \& Beesley, T. (2015). When goals conflict with values: counterproductive attentional and oculomotor capture by reward-related stimuli. Journal of Experimental Psychology. General, 144(1), 158-171. https://doi.org/10.1037/ xge0000037

Liang, F., Paulo, R., \& Molina, G. (2008). Mixtures of g Priors for Bayesian Variable Selection. Journal of the American Statistical Association, 103(481), 410-423. https://doi.org/10.1198/ 016214507000001337

Lorca-Puls, D. L., Gajardo-Vidal, A., White, J., Seghier, M. L., Leff, A. P., Green, D. W., Crinion, J. T., Ludersdorfer, P., Hope, T. M. H., Bowman, H., \& Price, C. J. (2018). The impact of sample size on the reproducibility of voxel-based lesion-deficit mappings. Neuropsychologia, 115, 101-111. https://doi.org/10.1016/j. neuropsychologia.2018.03.014

Milstein, D. M., \& Dorris, M. C. (2007). The influence of expected value on saccadic preparation. The Journal of Neuroscience: The Official Journal of the Society for Neuroscience, 27(18), 4810-4818. https:// doi.org/10.1523/JNEUROSCI.0577-07.2007

Moore, T., \& Zirnsak, M. (2017). Neural Mechanisms of Selective Visual Attention. Annual Review of Psychology, 68, 47-72. https://doi.org/ 10.1146/annurev-psych-122414-033400

Morey, R. D., Rouder, J. N., \& Jamil, T. (2014). BayesFactor: Computation of Bayes factors for common designs. $R$ Package Version 0. 9, 8

Morgenstern, O., \& Von Neumann, J. (1953). Theory of Games and Economic Behavior. Princeton, NJ. Princeton University Press

Nickerson, R. S. (2000). Null hypothesis significance testing: a review of an old and continuing controversy. Psychological Methods, 5(2), 241-301. https://www.ncbi.nlm.nih.gov/pubmed/10937333

Nothdurft, H. (2000). Salience from feature contrast: additivity across dimensions. Vision Research, 40(10-12), 1183-1201. https://doi. org/10.1016/S0042-6989(00)00031-6

Padoa-Schioppa, C., \& Assad, J. A. (2008). The representation of economic value in the orbitofrontal cortex is invariant for changes of menu. Nature Neuroscience, 11(1), 95-102. https://doi.org/10. $1038 / \mathrm{nn} 2020$

Pelli, D. G. (1997). The VideoToolbox software for visual psychophysics: transforming numbers into movies. Spatial Vision, 10(4), 437 442. http://www.ncbi.nlm.nih.gov/pubmed/9176953

Platt, M. L., \& Glimcher, P. W. (1999). Neural correlates of decision variables in parietal cortex. Nature, 400(6741), 233-238. https:// doi.org/10.1038/22268

Posner, M. I. (1980). Orienting of attention. The Quarterly Journal of Experimental Psychology, 32(1), 3-25. https://doi.org/10.1080/ 00335558008248231

Prinzmetal, W., Whiteford, K. L., Austerweil, J. L., \& Landau, A. N. (2015). Spatial attention and environmental information. $J$ Exp Psychol Hum Percept Perform, 41(5), 1396-1408. https://doi.org/ 10.1037/a0039429

Raymond, J. E., \& O'Brien, J. L. (2009). Selective Visual Attention and Motivation. Psychological Science. https://doi.org/10.1111/j.14679280.2009.02391.x

R Core Team. (2013). The R project for statistical computing. Available at Www.R-Project.Org/. Accessed October.

Roelfsema, P. R., Lamme, V. A. F., \& Spekreijse, H. (2000). The implementation of visual routines. Vision Research, 40(10), 1385-1411. https://doi.org/10.1016/S0042-6989(00)00004-3
RStudio Team. (2016). RStudio: Integrated Development Environment for $R$. RStudio, Inc. http://www.rstudio.com/

Schmitz, T. W., \& Duncan, J. (2018). Normalization and the Cholinergic Microcircuit: A Unified Basis for Attention. Trends in Cognitive Sciences, 22(5), 422-437. https://doi.org/10.1016/j.tics.2018.02. 011

Serences, J. T. (2008). Value-based modulations in human visual cortex. Neuron, 60(6), 1169-1181. https://doi.org/10.1016/j.neuron.2008. 10.051

Sha, L. Z., \& Jiang, Y. V. (2016). Components of reward-driven attentional capture. Attention, Perception \& Psychophysics, 78(2), 403414. https://doi.org/10.3758/s13414-015-1038-7

Shannon, C. E. (1948). A Mathematical Theory of Communication. Bell System Technical Journal, 27(3), 379-423. https://doi.org/10.1002/ j.1538-7305.1948.tb01338.x

Stănişor, L., van der Togt, C., Pennartz, C. M. A., \& Roelfsema, P. R. (2013). A unified selection signal for attention and reward in primary visual cortex. Proceedings of the National Academy of Sciences of the United States of America, 110(22), 9136-9141. https://doi. org/10.1073/pnas.1300117110

Stankevich, B. A., \& Geng, J. J. (2014). Reward associations and spatial probabilities produce additive effects on attentional selection. Attention, Perception \& Psychophysics, 76(8), 2315-2325. https:// doi.org/10.3758/s13414-014-0720-5

Sternberg, S. (1998). Discovering mental processing stages: The method of additive factors. The MIT Press. http://psycnet.apa.org/psycinfo/ 1999-02657-014

Vossel, S., Mathys, C., Stephan, K. E., \& Friston, K. J. (2015). Cortical Coupling Reflects Bayesian Belief Updating in the Deployment of Spatial Attention. The Journal of Neuroscience: The Official Journal of the Society for Neuroscience, 35(33), 11532-11542. https://doi.org/10.1523/JNEUROSCI.1382-15.2015

Vossel, S., Thiel, C. M., \& Fink, G. R. (2006). Cue validity modulates the neural correlates of covert endogenous orienting of attention in parietal and frontal cortex. NeuroImage, 32(3), 1257-1264. https://doi. org/10.1016/j.neuroimage.2006.05.019

Wagenmakers, E.-J. (2007). A practical solution to the pervasive problems ofp values. Psychonomic Bulletin \& Review, 14(5), 779-804. https://doi.org/10.3758/BF03194105

Yee, D. M., \& Braver, T. S. (2018). Interactions of Motivation and Cognitive Control. Current Opinion in Behavioral Sciences, 19, 83-90. https://doi.org/10.1016/j.cobeha.2017.11.009

Yin, H. H., \& Knowlton, B. J. (2006). The role of the basal ganglia in habit formation. Nature Reviews. Neuroscience, 7(6), 464-476. https://doi.org/10.1038/nrn1919

Zhao, Q., \& Koch, C. (2012). Learning visual saliency by combining feature maps in a nonlinear manner using AdaBoost. Journal of Vision, 12(6), 22. https://doi.org/10.1167/12.6.22

Open Practices Statement The data and materials for both experiments are available online. See: https:/github.com/kel-github/attention-valuecertainty

Publisher's note Springer Nature remains neutral with regard to jurisdictional claims in published maps and institutional affiliations. 